\title{
Long-term balancing selection contributes to adaptation in Arabidopsis and its relatives
}

\author{
Qiong Wu', Ting-Shen Han ${ }^{1,2}$, Xi Chen ${ }^{1,2}$, Jia-Fu Chen ${ }^{1,2}$, Yu-Pan Zou ${ }^{1,2}$, Zi-Wen Li ${ }^{1}$, Yong-Chao Xu ${ }^{1,2}$
} and Ya-Long Guo ${ }^{1,2^{*}}$ (D)

\begin{abstract}
Background: In contrast to positive selection, which reduces genetic variation by fixing beneficial alleles, balancing selection maintains genetic variation within a population or species and plays crucial roles in adaptation in diverse organisms. However, which genes, genome-wide, are under balancing selection and the extent to which these genes are involved in adaptation are largely unknown.

Results: We performed a genome-wide scan for genes under balancing selection across two plant species, Arabidopsis thaliana and its relative Capsella rubella, which diverged about 8 million generations ago. Among hundreds of genes with shared coding-region polymorphisms, we find evidence for long-term balancing selection in five genes: AT1G35220, AT2G16570, AT4G29360, AT5G38460, and AT5G44000. These genes are involved in the response to biotic and abiotic stress and other fundamental biochemical processes. More intriguingly, for these genes, we detected significant ecological diversification between the two haplotype groups, suggesting that balancing selection has been very important for adaptation.

Conclusions: Our results indicate that beyond the well-known S-locus genes and resistance genes, many loci are under balancing selection. These genes are mostly correlated with resistance to stress or other fundamental functions and likely play a more important role in adaptation to diverse habitats than previously thought.
\end{abstract}

Keywords: Adaptation, Arabidopsis thaliana, Balancing selection, Capsella rubella, Trans-species polymorphism

\section{Background}

Understanding the maintenance of genetic variation in the face of genetic drift is of critical importance to decipher the mechanisms of adaptation. Balancing selection maintains advantageous genetic variation within a population [1-6] and has received long-term attention in evolutionary biology [7]. Classical examples are mainly restricted to the major histocompatibility locus $(\mathrm{MHC})$ in vertebrates [8], $\mathrm{ABO}$ blood group in primates [5], heterokaryon incompatibility in fungi [9], and selfincompatibility (S) loci [10-13] and disease resistance (R) genes [14] in plants. Recently, a novel gene involved in resistance to severe malaria was found in a region of

\footnotetext{
* Correspondence: yalong.guo@ibcas.ac.cn

'State Key Laboratory of Systematic and Evolutionary Botany, Institute of

Botany, Chinese Academy of Sciences, Beijing 100093, China

2University of Chinese Academy of Sciences, Beijing 100049, China
}

ancient balancing selection in a genome-wide association study [15]. In another case, balancing selection on the srx-43 locus in Caenorhabditis elegans [16] surprisingly shapes density-dependent foraging behaviors. Obviously, almost all loci under balancing selection are important for the fitness of organisms.

Despite rapid progress in the understanding of balancing selection, confirmed cases are rather limited. Therefore, a question naturally follows, namely, what other genes in the genome are under balancing selection? The availability of genome-wide variation data provides the opportunity to comprehensively address this question.

One convenient signature of ancient balancing selection is trans-species polymorphisms (TSPs) [2, 17], i.e. ancestral polymorphisms that arose before species diverged from a common ancestor, survived the split, and segregate in the present populations of different species. 
However, TSPs can be neutral if there is not enough time for genetic drift to erase the ancestral polymorphisms in any species. In contrast, if divergence time is sufficiently long, neutral TSPs will be eliminated and only the ones under balancing selection can survive. The surrounding ancestral regions of TSPs under balancing selection may be broken up by recombination, which becomes narrower as balancing selection becomes more ancient $[5,18]$. Furthermore, TSPs can be easily confounded with recurrent mutations or introgression (Fig. 1), since both of the cases also produce shared polymorphisms in closely related species [17]. Nevertheless, some features are associated with ancient balancing selection [18]. In particular, orthologous sequences from different species cluster by allele, rather than species, due to tight linkage around the site under balancing selection $[3,18]$, while neutral recurrent shared polymorphisms still cluster by species. Another associated signal of long-term balancing selection is a high level of variation, since the polymorphisms surrounding TSPs should be more ancient than the genome-wide average coalescent time. Furthermore, the allele frequency distribution for sites under balancing selection is expected to exhibit a trend towards intermediate frequencies [18].

Genome-wide scans for TSPs are rather limited to Hominidae-related species [3, 19], except for one recent study in plants [20], in which a complex history of nonbifurcating speciation was identified in the genus Arabidopsis and many ancestral polymorphisms were detected. However, it is not clear whether these ancestral polymorphisms are maintained by balancing selection.

In this study, we performed a whole-genome scan for TSPs under balancing selection between two plant species, Arabidopsis thaliana and Capsella rubella. Unless otherwise specified, TSPs in this study refer to those under balancing selection, not neutral ones. Arabidopsis and Capsella belong to Brassicaceae and diverged about 8 million years (or $\sim 8$ million generations) ago [21], a much larger timescale than that of previous studies of humans and chimpanzees ( 0.25 million generations) $[3,5,19]$. Beyond this, C. rubella, a highly selfing species, recently diverged from its outcrossing relative Capsella grandiflora and has experienced a severe population bottleneck, resulting in a tremendous reduction in genetic diversity and ancestral polymorphisms [22-24]. Therefore, TSPs that persist for such a long time, particularly through the dramatic demographic event, must be of critical importance for plants. We are particularly interested to see, as a kind of standing variation, the extent to which polymorphisms at loci under balancing selection could contribute to adaptation.

Interestingly, we detected 433 candidate genes that exhibit shared coding single nucleotide polymorphisms

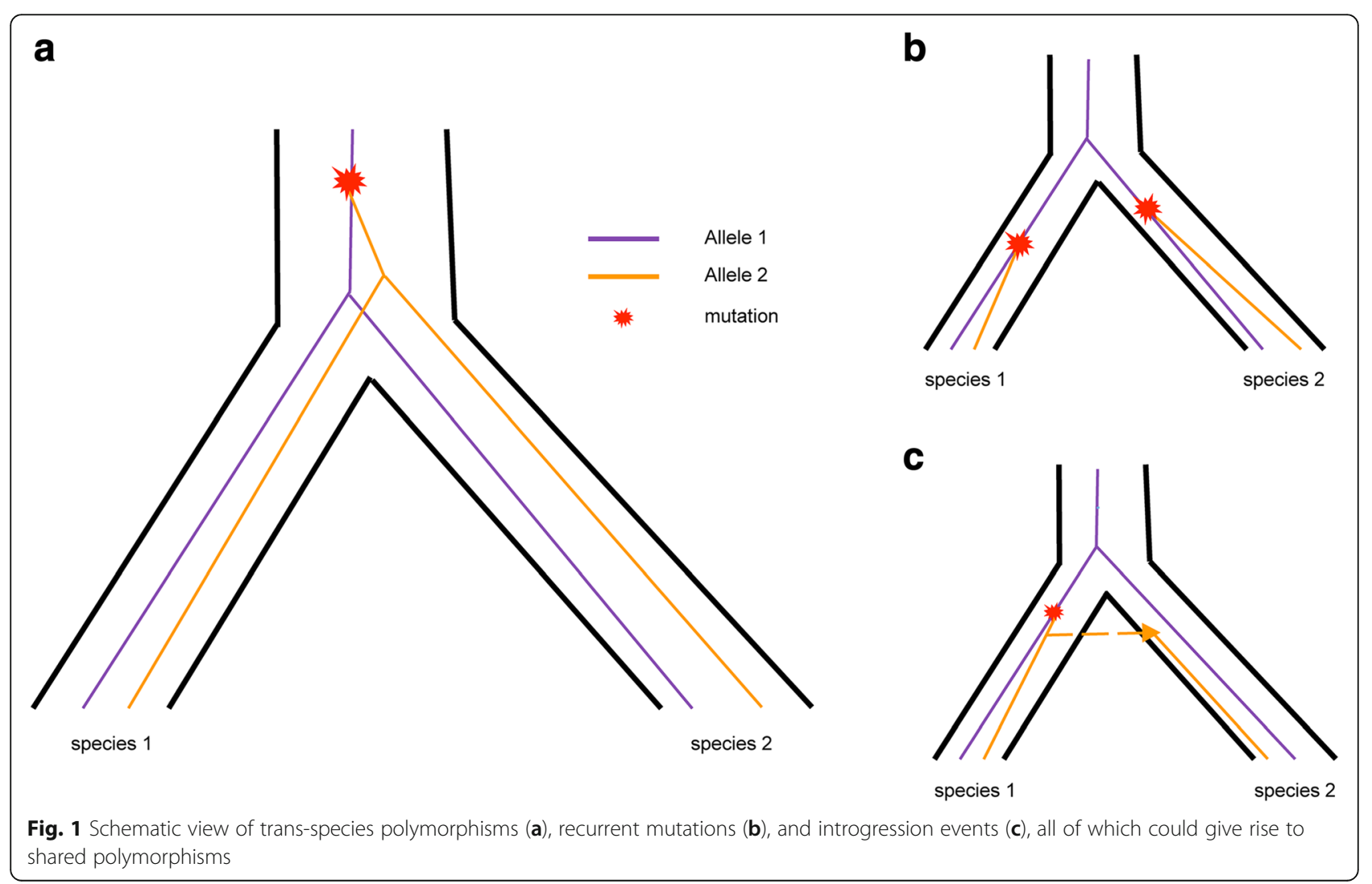


(SNPs) between the two species. We confirmed that five of these genes were under long-term balancing selection. Furthermore, there was significant ecological diversification between the two haplotype groups separated by the TSP sites. Our results indicate that, in plants, beyond the well-known $\mathrm{S}$-locus genes and $\mathrm{R}$ genes, many loci are under balancing selection. These genes were mostly associated with resistance to stress and other fundamental functions and likely play an important role in adaptation to diverse habitats.

\section{Results}

Shared polymorphisms are abundant between $A$. thaliana and C. rubella

In a population of $80 \mathrm{~A}$. thaliana accessions [25], there were 4,902,039 SNPs (out of 119,146,348 sites), among which 2,044,731 had a minor allele frequency (MAF) of $>0.05$. In the C. rubella population, by calling SNPs from 22 C. rubella accessions (Additional file 1: Table S1, including 21 published accessions [26] and one accession sequenced in this study [27]) against the C. rubella reference genome [28], we identified 2,149,643 SNPs (out of $134,834,574$ sites), of which 1,240,547 had a MAF $>0.05$. To identify shared polymorphisms between the two species, defined as the same allele pair at a particular orthologous site, we first constructed the set of orthologous gene pairs between the two species. To guarantee that the orthologous genes are conserved, in addition to the reference genomes of $A$. thaliana and $C$. rubella, we included Arabidopsis lyrata [29], a congener of $A$. thaliana. We got 16,047 orthologous gene pairs and removed 33 that had tandem duplications in any of the three references and finally obtained a total of 16,014 orthologous gene pairs between $A$. thaliana and $C$. rubella for further analysis.

The genic region of the 16,014 orthologous genes in $A$. thaliana spanned $39,275,210$ bp and similarly, in C. rubella, it spanned 40,936,262 bp. These regions contained $3,889,495$ fixed differences and this high ratio $(\sim 10 \%)$ is consistent with the long divergence time ( 8 MYA) of the two species [21]. In these regions, we found $1,122,845$ bi-allelic sites $(426,123$ with MAF $>0.05)$ in A. thaliana and 452,116 bi-allelic sites $(279,780$ with MAF $>0.05)$ in C. rubella. Among these polymorphic sites, 19,732 orthologous sites were polymorphic in both species, of which 8535 shared the same allele pair (shared SNP [shSNP]) (Additional file 1: Table S2).

Compared with non-coding region sequences, coding region sequences are more conserved and yield robust alignments between the two highly diverged species; therefore, we first focused on shSNPs in coding regions. MAF $>0.05$ was required in both species to guarantee SNP reliability and account for the expected excess of alleles with intermediate frequencies for sites under long-term balancing selection. We found 1503 shSNPs in the coding regions of 1007 genes.

Further filtering was applied to the 1503 shSNPs to avoid genotyping and mapping errors. The filtering was only applied to the C. rubella SNP data, since we downloaded the SNP matrix for A. thaliana. To avoid spurious SNPs incurred by duplications in the genome, we assessed the mappability of every 50-bp region in C. rubella and only retained sites that were in uniquely mappable regions for subsequent analysis. This left only 580 sites. Finally, after removing low-quality sites marked by the SNP calling tool, we obtained 546 reliable shared coding SNPs in 433 genes. Details of the filtering process can be found in the "Methods" section and a view of the process is depicted in Fig. 2.

\section{Demographic history of the two species}

The detection of real TSP signals from the abundant shared polymorphisms relies on a full understanding of the demographic history of the two species. The joint site frequency spectrum (joint SFS) has been widely used to study the demographic history of diverse organisms $[30,31]$. Therefore, we first extracted the fourfold degenerate sites from the alignments of the reference genomes of A. thaliana and C. rubella on the 16,014 orthologues. Finally, we obtained 2,011,573 sites for the demographic analysis (see "Methods" for details).

Coalescence simulations were then run using fastsimcoal2 [30] under a basic model without gene flow (M1, Fig. 3) and a model incorporating ancient gene flow between the two genera (M2, Fig. 3). We considered only ancient gene flow between the two species, since species belonging to different genera and with different numbers of chromosomes (five vs eight) are highly unlikely to have recent introgression. In addition, in both genera, $A$. thaliana is the only species with five rather than eight chromosomes [32]; we therefore restricted the ancient gene flow before $A$. thaliana separated from the rest of the Arabidopsis genus. In each model, we set the divergence time of the two genera to 8 MYA [21], which amounts to 8 million generations ago, and assumed a spontaneous mutation rate of $7 \times 10^{-9}$ per bp per generation [33]. We considered various population sizes for both species based on the transition events from their respective progenitors; $A$. thaliana underwent a population reduction after it diverged from the rest of the Arabidopsis genus around 6 MYA [21] and C. rubella experienced a very recent bottleneck associated with the speciation from C. grandiflora [23, 24]. We used coalescent simulations applying the composite likelihood method implemented in fastsimcoal2 [30] to fit both models to the joint SFS of the two species computed from the extracted 2,011,573 trans-species fourfold degenerate sites. We compared the two models using 
80 accessions of Ath 4,902,039 SNPs

22 accessions of Cru 2,149,643 SNPs

Restrict to 16014 orthologous genes

8535 shared SNPs in genic region

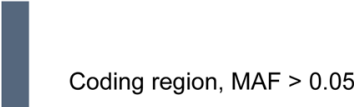

1503 shared coding SNPs

Filters to further remove potential genotyping or mapping errors based on mappability and quality

546 reliable shared coding SNPs lying on 433 candidate genes

Window scanning across each gene (the whole genic region, covering each of the 975 shard SNPs with MAF>0.05 on these 433 genes, intronic or exonic), to look for 100 bp aligned windows that cover at least 2 of the 975 shared SNPs that are in strong LD and cluster by allele

\section{0 sites on 5 final candidate genes}

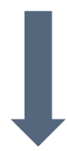

Verified by sanger sequencing

Fig. 2 Pipeline of the SNP filtering process to identify candidate TSP sites

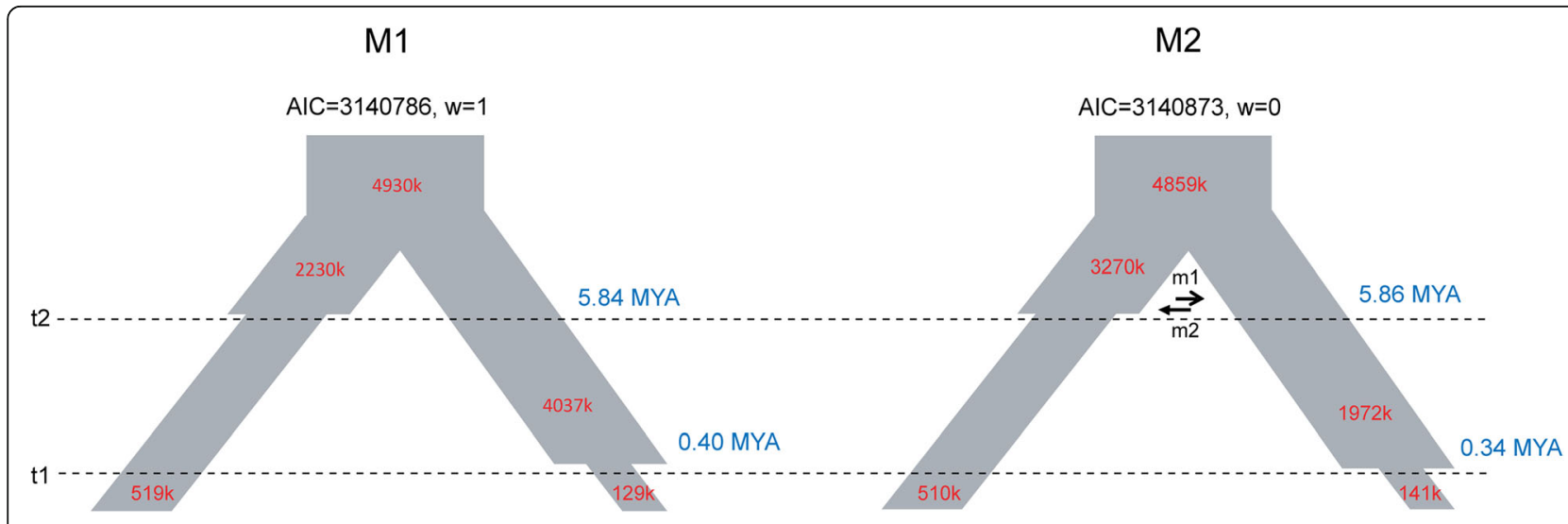

Fig. 3 Demographic parameter estimates for two models of the divergence of the two species 
Akaike's information criterion (AIC) and Akaike's weight of evidence (w), as in Excoffier et al. [30]. The model without ancient gene flow (M1) fit slightly better (Max EstLhood: -682010 vs -682028$)$, with a lower AIC and higher weight than those of the other model (Fig. 3, Additional file 2: Table S3). In addition, the two close likelihoods indicate that the effect of ancestral gene flow should have been wiped out over the long time scale and contributes little to model quality.

Under Model M1, the current $N_{\mathrm{e}}$ of $A$. thaliana was 519,000 with a $95 \%$ confidence interval (CI) of $486,368-$ 527,574 , from a large ancestral population $(\sim 2,230,000$, $95 \% \mathrm{CI}=1,085,330-4,876,051)$ before separating from the rest of the Arabidopsis genus at 5.84 MYA (95\% $\mathrm{CI}=5.27-6.70)$. C. rubella evolved $~ 0.40$ MYA (95\% CI $=321,998-500,317)$ from an ancestral population with a large $N_{\mathrm{e}}$ of $\sim 4,037,000 \quad(95 \% \mathrm{CI}=2,076,868-5,165,614)$ and a current $N_{\mathrm{e}}$ of $\sim 129,000 \quad(95 \% \quad \mathrm{CI}=126,383-$ 157,779). The two genera diverged from an ancestral population with $N_{\mathrm{e}}=\sim 4,930,000(95 \% \mathrm{CI}=4,560,931-$ 4,969,696). Under Model M2 with gene flow, similar parameter estimates were obtained, except for a larger ancestral $N_{\mathrm{e}}$ for the Arabidopsis genus ( 3,270,000, 95\% CI $=797,016-4,342,346)$ and a smaller $N_{\mathrm{e}}$ for the Capsella genus ( 1,972,000, 95\% CI = 2,126,346-6,248,003). Stronger gene flow was estimated from Capsella to Arabidopsis than in the reverse direction (migration rate per generation; $1 \times 10^{-8}, 95 \% \mathrm{CI}=4.0 \times 10^{-15}-1.1 \times 10^{-6}$ vs $\left.7 \times 10^{-14}, 95 \% \mathrm{CI}=5.7 \times 10^{-15}-6.1 \times 10^{-5}\right)$, although both were weak (see Additional file 2: Table S3 for the details).

\section{Trans-species polymorphisms between the two species must be under balancing selection}

Trans-species polymorphisms can be neutral and its probability can be approximated given specific demographic parameters. Similar to a study of TSPs in humans and chimpanzees [34], under neutral evolution, shared polymorphisms were identical by descent in our system only if: (1) at least two $A$. thaliana lineages and two C. rubella lineages did not coalesce before the $A$. thaliana-C. rubella split; and (2) lineages carrying the same allele coalesced before lineages carrying different alleles. This probability is mainly determined by condition (1) and can be approximated by the following based on the coalescent theory [34]:

$$
P=e^{-\frac{T}{2 N_{A}}} * e^{-\frac{T}{2 N_{C}}},
$$

where $T$ refers to the divergence time of the two genera and $N_{\mathrm{A}} / N_{\mathrm{C}}$ refers to the population sizes of $A$. thalianal C. rubella, respectively. According to our estimates under Model M1, taking population size changes into consideration, this probability of identity by descent is on the order of $10^{-9}$. Given that we have $<39,275,210$ aligned sites between the two species in genic region, we expect the total number of neutral TSPs to be $<1$ by genetic drift alone.

We assumed random mating in our model; however, both species are selfing and population structure probably exists within species. Nevertheless, recent demographic events should have relatively little effect, since we require deep coalescence events by chance in both species in the same region of the genome $[3,18]$. As illustrated in previous study [3], even deep population structure within modern humans should have minimal effect on the probability. In this study, both species have a history of predominantly outcrossing. A. thaliana transitioned from outcrossing to selfing only one million years ago [35] and C. rubella transitioned much more recently [23, 24]. Even as selfing species, the outcrossing rate of local populations is as high as $14.5 \%$ [36]. Therefore, population structures, if existing, are unlikely to persist over a long time scale and its impact on the probability can thus be ignored.

\section{Identification of trans-species polymorphisms under bal- ancing selection}

TSPs can be distinguished from neutral mutations because regions under long-term balancing selection cluster by allele, rather than by species [18]. Therefore, we next focused on the 433 candidate genes with reliable shared SNPs in the coding region and examined the haplotypes covering each shared bi-allelic SNP with MAF $>0.05$ in the genic regions.

To estimate the length of each segment carrying a signal of TSPs, we used a formula derived previously [5] that relies largely on the recombination rate. From a coalescence point of view, such a segment is not broken up by recombination until all of the lineages from the same allelic class coalesce to their most recent common ancestor in the ancestral population [18]. Adopting a recombination rate of $3.6 \mathrm{cM} / \mathrm{Mb}$ [37] for both species, the length of the segment was extremely short, i.e. only several base pairs, theoretically. Given that both species recently arose from their respective outcrossing progenitors $[23,24,35]$ and the effective recombination rate could be much higher in the past, the expected length may be even shorter. This estimate suggests, under the neutral circumstances in our system, that it is highly difficult to discover any segment without a break from recombination. However, when balancing selection exists, selection can suppress recombination in the surrounding region [34]. Therefore, the segment length should be longer than the theoretical estimated under a neutral model. We thereby scanned the genic region using a window size of $100 \mathrm{bp}$ and a 1-bp step size. 
In the 433 candidate genes, we detected 975 shared biallelic SNPs (including both exonic and intronic SNPs with MAF > 0.05). Similar to previous studies [3, 19, 20], we next looked for windows covering at least two of the 975 SNPs that are in strong linkage disequilibrium $\left(r^{2}>\right.$ 0.5 ) in both species among the qualified windows (aligned at a minimum of $95 \%$ of the length; see "Methods" for details) to identify allelic trees. These restrictions can greatly reduce false positives and yield allelic trees, if they exist, with high resolution. Finally, we identified windows from five genes, AT1G35220, AT2G16570, AT4G29360, AT5G38460, and AT5G44000, involving ten sites, as candidate TSPs under long-term balancing selection (Additional file 3: Figure S1). None of the five orthologous genes we found here are correlated with copy number variation (CNV) and all of them have only one hit when we compared them against the references of the two species, respectively (see "Methods" for details).

To verify the regions identified, we first determined all haplotypes in the identified regions from each population and resequenced representative accessions for each haplotype [38, 39] (see Additional file 1: Table S4 for the primers). As expected, all of the candidate TSP sites in the five genes were validated and the sequences of the two species in the candidate regions clustered by allele, rather than species (Fig. 4). In the gene AT1G35220, the two candidate TSP sites were in complete linkage disequilibrium in an intronic region; this region may be the target of balancing selection or linked to an undetected coding TSP site.

Although the haplotypes of each region clustered by allele, rather than species, haplotype sharing between the two species was rarely detected, except in AT2G16570 (Col-0 shared its haplotype with several $C$. rubella accessions; Fig. 4). This is not surprising given the long divergence time; extensive haplotype sharing usually appears at a much smaller timescale and is induced by events such as recent introgression between closely related species.

\section{Neutral simulation studies validate the five candidate genes}

To see if the observed windows could be generated randomly under neutral evolution, resulting in false positives, we ran additional simulations based on the estimated demographic parameters using fastsimcoal2 [30] (Additional file 4: Text S1). Apart from neutral recurrent mutations, gene flow can also result in shared SNPs. Accordingly, we ran simulations under both Model M1 (without gene flow) and M2 (with ancient gene flow), although our demographic analysis indicated that M1 slightly better fit the data. In both simulations, we considered heterogeneity in mutation rates for different classes of mutations, notably the higher mutation rate at $\mathrm{CpG}$ sites, which may result in false positives (Additional file 1: Table S5, Additional file 4: Text S1). Using fastsimcoal2 [30], we generated 1,000,000 neutral segments of $100 \mathrm{bp}$ under each model and looked for those with two or more shared SNPs and cluster by allele as we searched for TSPs.

For both models, none of the 1,000,000 runs gave rise to a window that met our criteria (Additional file 1: Table S6). Despite existence of neutral shared SNPs, no simulated window rendered an allelic tree, since all the windows with shared SNPs were accompanied by much more fixed differences between the two species, implying higher divergence levels than diversity. This result suggest that these simulated neutral shared SNPs are recurrent mutations, rather than TSPs, and more importantly, the five genes we found are not consistent with neutral evolution and thereby proved to be real TSPs under balancing selection. The final TSP sites and genes are listed in Table 1. Furthermore, together with the aforementioned demographic study, our results imply that even if ancient gene flow occurred, under neutral evolution, TSPs would be lost by drift in this system.

\section{Properties of the genes under balancing selection}

We next calculated nucleotide diversity $(\pi)$ for all TSP regions in the five genes in each species and used the simulated neutral sequences under M1 to determine background diversity levels. All of the regions in the five genes exhibited significantly higher $\pi$ values than background levels in both $C$. rubella and A. thaliana (WilcoxonMann-Whitney test, FDR-corrected $P<0.05$, Table 2, Additional file 3: Figure S2A), except AT5G38460 in A. thaliana. In addition, the alleles of these genes showed a trend towards intermediate frequencies (WilcoxonMann-Whitney test, $P=0.0752 / 0.03474$ for $A$. thalianal C. rubella; Additional file 3: Figure S2B). However, an intermediate frequency is an indication of balancing selection, but not definitive evidence, since the allele frequency distribution of sites linked to a balanced polymorphism is expected to exhibit a shift towards the frequency equilibrium, which can be at any allele frequency [19].

One of the five genes under long-term balancing selection in this study, AT1G35220, has an unknown function, but exhibits protein phosphorylation under ethylene treatment [40]. Among others, AT2G16570 is a key enzyme in the purine nucleotide biosynthesis pathway and is important for cell division, chloroplast biogenesis, and seed germination [41, 42]; AT4G29360 is an $O$-glycosyl hydrolase family 17 protein, involved in defense responses [43]; AT5G38460 is a glycosyltransferase and it catalyzes the transfer of a glycosyl group from one compound (donor) to another (acceptor) and is involved in diverse functions, including biotic stress [44]; AT5G44000 is a glutathione $S$-transferase, which is 


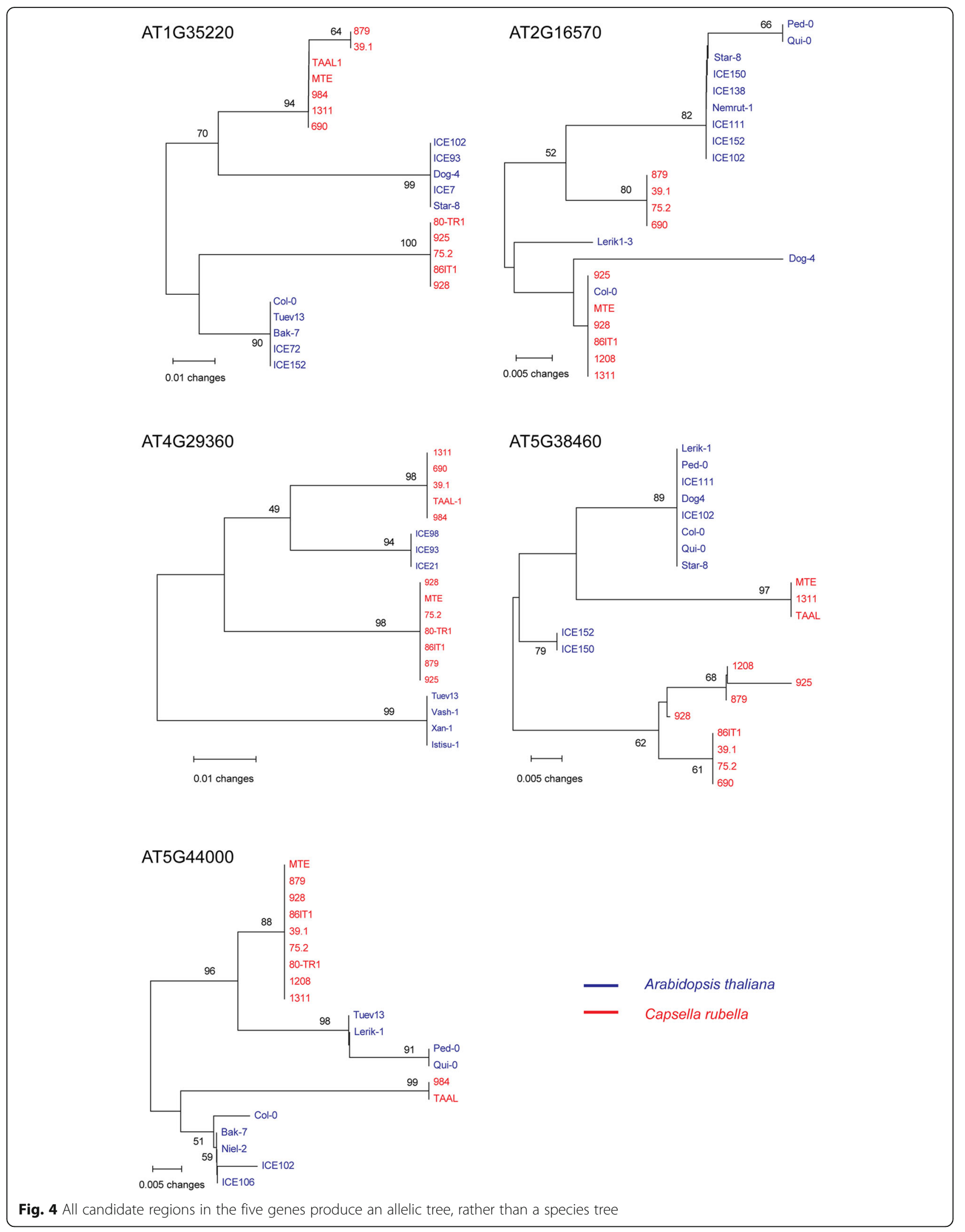




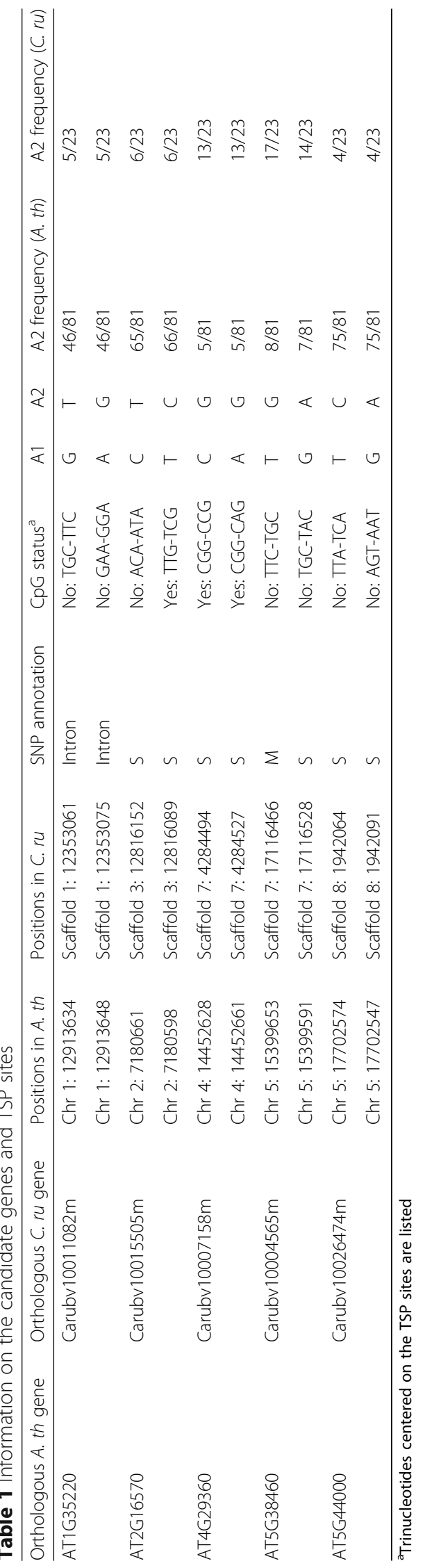


Table 2 Genetic features of TSP sites

\begin{tabular}{llllll}
\hline Orthologous gene $($ A. th $)$ & Site label & Position in A. th & $r^{2}($ A. th/C. ru $)$ & $\pi^{a}($ A. th/C. ru $)$ & MAF $($ A. th/C. ru $)$ \\
\hline AT1G35220 & TSP-1 & Chr 1: 12913634 & $1 / 1$ & $0.036(<2.2 \mathrm{E}-16) / 0.037(<2.2 \mathrm{E}-16)$ & $0.432 / 0.217$ \\
& TSP-2 & Chr 1: 12913648 & & & $0.432 / 0.217$ \\
AT2G16570 & TSP-3 & Chr 2: 7180661 & $0.92 / 1$ & $0.017(2.9 \mathrm{E}-9) / 0.019(5.9 \mathrm{E}-10)$ & $0.198 / 0.261$ \\
& TSP-4 & Chr 2: 7180598 & & $0.009(1.8 \mathrm{E}-4) / 0.034(<2.2 \mathrm{E}-16)$ & $0.185 / 0.261$ \\
AT4G29360 & TSP-5 & Chr 4: 14452628 & $1 / 1$ & $0.062 / 0.434$ \\
& TSP-6 & Chr 4: 14452661 & & $0.007(0.34) / 0.031(6.4 \mathrm{E}-3)$ & $0.062 / 0.434$ \\
AT5G38460 & TSP-7 & Chr 5: 15399653 & $0.55 / 0.86$ & & $0.099 / 0.261$ \\
& TSP-8 & Chr 5: 15399591 & & & $0.086 / 0.391$ \\
AT5G44000 & TSP-9 & Chr 5: 17702574 & $1 / 1$ & $0.010(1.4 \mathrm{E}-10) / 0.023(<2.2 \mathrm{E}-16)$ & $0.074 / 0.174$ \\
& TSP-10 & Chr 5: 17702547 & & & $0.074 / 0.174$
\end{tabular}

${ }^{a}$ Values are the averages of the corresponding values in all $100 \mathrm{bp}$ windows covering the TSP sites in strong linkage disequilibrium that could yield an allelic tree, and their associated FDR-adjusted $P$ values are listed in parentheses

usually involved in the response to abiotic and biotic stress [45]. Apparently, these genes are potentially involved in the response to biotic or abiotic stress (AT4G29360, AT5G38460, and AT5G44000) or fundamental biochemical functions (AT2G16570).

As expected, the genes under balancing selection were functionally important and all of the homologues of the five genes already existed in the most recent common ancestor of green plants. As indicated in Table S7 (Additional file 1: Table S7), homologues (either orthologues or paralogues) can be found even in the most basal species of green plants, Chlamydomonas reinhardtii, for all of the five genes, except AT4G29360, which can be traced back to Physcomitrella patens.

However, loci that are widely accepted to be under balancing selection, such as the S-locus [10] or R genes [14], did not stand out in this study. This is expected, since these loci are too variable to identify based on short reads. For example, $\mathrm{R}$ genes are too dynamic to call SNPs [46]; the S-locus does not exist in the latest annotation of the Arabidopsis genome and only one Slocus haplotype is maintained in C. rubella since the transition from outcrossing to selfing and the breakdown of self-incompatibility [24]. Furthermore, the S-locus is no longer under balancing selection, since both species are now selfing. In contrast, the genes we identified here, although ancient, have not been comprehensively studied and may provide insight into the types of genes under balancing selection.

\section{Balancing selection contributed to adaptation to divergent habitats}

To see if the allelic variants under long-term balancing selection are associated with ecological diversification, we investigated divergence with respect to 48 ecological factors (Additional file 5: Table S8A). Due to a lack of GPS information and the small sample size of C. rubella, this analysis was only possible for the A. thaliana samples. Population structure is usually highly correlated with ecological diversification and may therefore confound our results. We first checked whether any TSP site was correlated with population structure in the $A$. thaliana samples, although such structure does not affect the probability of observing the species tree of $A$. thaliana and C. rubella. Using ADMIXTURE [47], we found that the $80 \mathrm{~A}$. thaliana samples can be classified into two groups (Additional file 3: Figure S3; Additional file 6: Table S9) and only the allelic classifications of the two sites from the gene AT5G38460 are significantly correlated with the population structure (chi-square test, FDR-corrected $P<0.05$; Additional file 1: Table S10). We thereby excluded AT5G38460 from subsequent ecological analyses.

To gain a thorough understanding of ecological divergence, we used 1135 recently published $A$. thaliana genomes [48]. First, we applied a "thinning" process to guarantee that every sample was highly representative of its natural habitat, which left 584 samples (see "Methods"). Second, for each gene, we classified the 584 accessions of A. thaliana into two groups based on the phased haplotypes for the two TSP sites (Additional file 5: Table S8B, $\mathrm{C}$, some samples were removed because they could not be phased). We then evaluated divergence between the two groups of accessions with respect to the 48 ecological factors for each of the four genes. Interestingly, all of these four genes were associated with the divergence of some specific ecological parameters. AT1G35220 and AT4G29360, in particular, exhibited significant divergence with respect to most of the temperature-related ecological factors (Additional file 5: Table S8 A, Wilcoxon-MannWhitney test, FDR-corrected $P<0.05$ ).

We next modeled the ecological niches for all four genes. Apparently, the two groups of samples for each gene, as indicated by Warren's I statistics that measures 
niche similarity [49], exhibited significantly lower observed niche identity than 100 random permutations (one-sample $t$-test, FDR-corrected $P<0.01$; Fig. 5a, Additional file 5: Table S8 D). In other words, the two allelic groups of samples exhibit significant niche divergence. Furthermore, the samples of each allelic type for each gene were scattered, instead of being isolated into a small local area (Additional file 3: Figure S4). These results suggest that all of these loci are correlated with adaptation.

We also examined expression differentiation for the four genes between the two corresponding groups based on the phased haplotypes at the two TSP sites choosing 84 published leaf-tissue-extracted transcriptomes from A. thaliana [50] (one sample was sequenced for each accession and the expression level was measured as fragments per kilobase of exon per million fragments mapped [FPKM]) as our previous study [51]. One gene, AT5G44000, exhibited significant expression difference (Wilcoxon-Mann-Whitney test, FDR-corrected $P<0.05$, Fig. 5b) between the two haplotype groups.

We therefore performed in-depth niche modeling of AT5G44000 (Fig. 5c) and examined the diversification of the two groups of samples (503 vs 75). We first compared the niche identity between the two haplotype groups of AT5G44000 by restricting our analysis to niches with a high probability $(\geq 0.5)$ and obtained similar results (Fig. 5c, Additional file 5: Table S8 D). To see if the unbalanced sample size could affect the results, we used another permutation strategy by restricting the analysis to the same sample size (75) for both sets in each repetition (with probability $>0.5$ ). As presented in

\begin{tabular}{llllll} 
a & \multicolumn{7}{l}{} \\
\cline { 2 - 6 } Gene & Type1 & Type2 & IO & IS & $P$ \\
\hline AT1G35220 & 123 & 433 & 0.901 & $0.939 \pm 0.010$ & $2.4 \mathrm{E}-62$ \\
AT2G16570 & 438 & 138 & 0.915 & $0.944 \pm 0.009$ & $1.9 \mathrm{E}-54$ \\
AT4G29360 & 18 & 562 & 0.718 & $0.851 \pm 0.030$ & $4.0 \mathrm{E}-66$ \\
AT5G44000 & 503 & 75 & 0.858 & $0.926 \pm 0.014$ & $6.8 \mathrm{E}-69$ \\
\hline
\end{tabular}

C
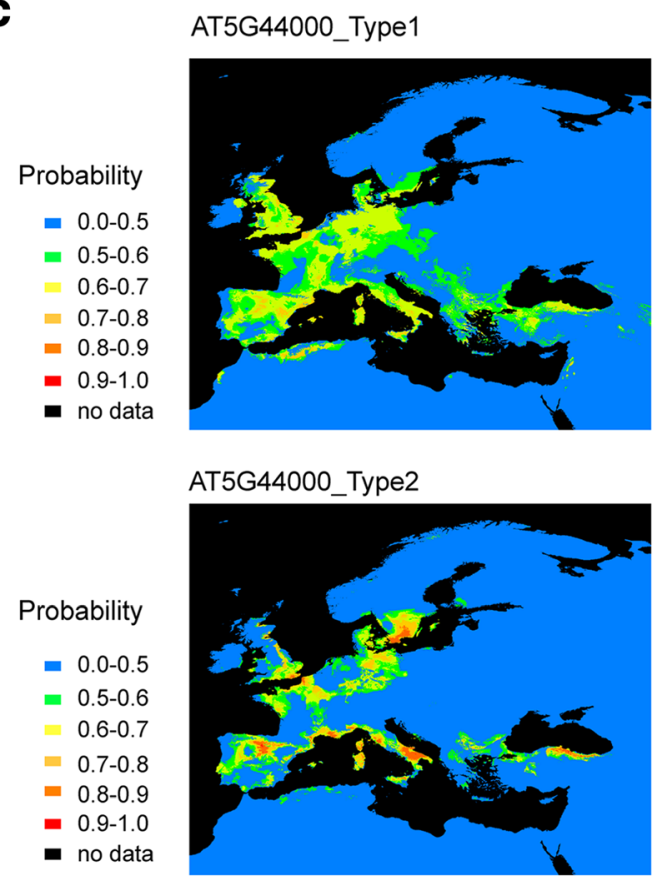

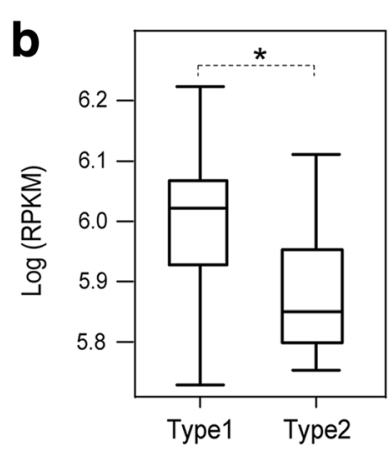

Histograms of I

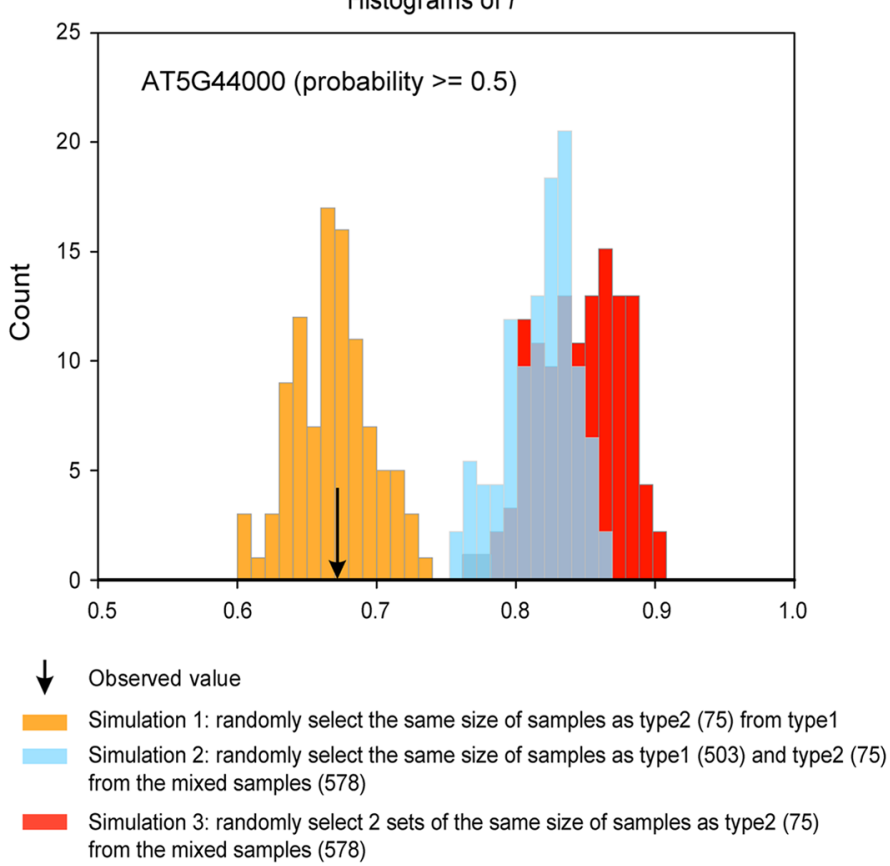

Fig. 5 Ecological and expression divergence. a Significant ecological divergence between the two types of samples for each of the four genes, as indicated by the observed / score $\left(I_{0}\right)$ and simulated / scores $\left(I_{\mathrm{s}}\right)$. b Expression divergence of the gene AT5G44000. c Left: Modeling of the niche with a high probability $(\geq 0.5)$ of the two types of samples for AT5G44000. Right: Significance results under different permutation strategies (for niches with probability $\geq 0.5 ; I_{O}=0.673,100$ permutations) 
Fig. 5c, when the permutation was performed for the real sample groups (simulation 1 ), the observed $I$ value (0.673) did not show a significant difference (one-sample $t$-test, $P=0.166$ ), indicating that the observed value was reliable, regardless of the sample size difference. When the two real groups were mixed and two random groups of real sizes were selected (simulation 2) or two random groups of equal size (75) were selected (simulation 3), the difference between the observed value and the permutations was significant again (one-sample $t$ test, $P=1.9 \times 10^{-75}$ for simulation 2 and $P=2.6 \times 10^{-75}$ for simulation 3 ). These results imply that the two functionally differentiated haplotype groups of AT5G44000 adapted to divergent ecological habitats.

\section{Discussion}

The importance of balancing selection for the maintenance of variation has received long-standing attention in evolutionary biology. However, many fundamental questions remain largely unanswered [3, 52]. For example, besides the well-known examples, what genes are under balancing selection across genomes, especially in plants? How long does genomic signature of balancing selection persist? In addition, as a kind of standing variation, to what extent does polymorphism of loci under balancing selection promote adaptation?

To address these questions, we performed a genomic scan for TSPs maintained in the model plant $A$. thaliana and its relative $C$. rubella, which diverged more than 8 million generations ago. TSPs can be neutral if the species diverged recently or have large effective population sizes. We therefore estimated demographic parameters for A. thaliana and C. rubella using population data and confirmed the theoretical prediction that neutral ancestral TSPs are highly unlikely in our system. After ruling out recurrent mutations and gene flow, we finally got five genes out of 433 candidate ones.

However, we focused on orthologous genes with two or more shared SNPs in both species, our method for TSP detection likely missed some genes with single TSP sites. Furthermore, we may have missed TSPs outside of genes; one previous study on humans and chimpanzees identified 123 non-coding TSPs and balancing selection has targeted regulatory variation in the human genome [3]. In addition, the further filters we used to get reliable shared coding SNPs may be too stringent, e.g. the mappability filter removed two-thirds of the shared SNPs. Nevertheless, this is a reasonable strategy to avoid false positives.

The genes under balancing selection may have played critical roles in adaptation to divergent habitats, since for four of the genes under balancing selection, the niches of the two haplotype groups were significantly differentiated. Unlike animals, in which genes experiencing balancing selection are mainly involved in resistance to viruses, in this study we found some other genes under balancing selection in plants, correlated with the response to stress and fundamental functions. In particular, these genes were correlated with diversifying niches, indicating that balancing selection probably has more influences on ecological distribution in plants than in animals. However, we cannot exclude the possibility that other environmental factors, especially microhabitat or biotic factors, like pathogens, contributed to the maintenance and spread of the genetic footprint of balancing selection [6]. More correlations may be discovered if additional factors are considered.

Loci under balancing selection are a good system to understand the mechanism of adaptation. The identification of genetic loci associated with both particular climatic factors and fitness traits is critical for the understanding of adaptive mechanisms in organisms, but this is extremely difficult to achieve given the limited time and usually weak selection strength [53, 54]. However, balancing selection can act through a variety of mechanisms $[1,2]$. For example, by temporal and spatial variation in selection [55], loci can be functionally associated with local or global environmental changes during their long-term evolutionary history. In this way, strong and clear selection signal could be kept in the genome and could be studied in-depth. For example, a recent study identified a novel locus of resistance to severe malaria [15], which was in a region of ancient balancing selection [3].

Finally, it will be interesting to determine how genetic differentiation at these loci contributes to adaptation to different habitats. Given that C. rubella experienced a severe population bottleneck during the breeding system transition [23, 24], TSPs that survived this dramatic demographic event could be of critical importance for plant fitness. Therefore, it will be highly rewarding to further investigate the biological functions of these five genes under ancient balancing selection or genes with candidate TSPs to determine the biological basis for balancing selection. Moreover, a genome scan for loci under balancing selection is an effective way to reveal functionally important genes or sequences that are difficult to detect using common reverse or forward genetics owing to weak or indirect effects on organisms.

\section{Conclusions}

We identified a number of shared polymorphic sites in the genomes of two closely related species and confirmed that at least five genes are under long-term balancing selection. The two different haplotypes of these genes under balancing selection were significantly 
differentiated with respect to habitats, suggesting that balancing selection could have contributed to adaptation to different habitats in plants in general.

\section{Methods}

\section{Species sampling and DNA sequencing}

The SNP matrix of 80 accessions of A. thaliana genomes (with ecotype Col-0 as the reference) [25] was downloaded from the Arabidopsis 1001 Genome Database (http://1001genomes.org/) and genome sequences of 21 C. rubella accessions were downloaded [26]. Together with another accession (928) sequenced here [27] and the reference accession MTE, 23 samples comprised the C. rubella population (see Additional file 1: Table S1 for a complete list of the C. rubella samples). Genomic DNA of accession 928 was extracted from seedlings using the CTAB method [56]. Paired-end sequencing libraries with an insert size of approximately $300 \mathrm{bp}$ were constructed. Then, 100-bp pair-end reads were sequenced using Illumina HiSeq 2000. To verify the sequences, partial fragments of the five candidate genes from representative ecotypes of C. rubella and A. thaliana were amplified and sequenced [38, 39]. Primers used for PCR and sequencing are listed in Additional file 1: Table S4. PCR products were sequenced using the ABI 3730 automated sequencer (Applied Biosystems, Foster City, CA, USA).

\section{Read processing, mapping, and genotyping}

Raw short reads were filtered using NGSQCToolkit v2.3.3 [57] with a cutoff PHRED quality score of 20 . The percentage of qualified nucleotide bases in a read should not be $<70 \%$. Every C. rubella sample was aligned to the reference MTE genome using BWA v0.7.12 [58] with default parameters allowing up to $4 \%$ mismatches and one gap. The reads were sorted, and duplicates were removed using the toolkit Picard v2.0.1 (http://broadinstitute.github.io/ picard/). The rest of the genotyping pipeline was performed using the toolkit GATK v3.5 [59]. Reads present in areas surrounding InDels were realigned using the built-in function IndelRealigner, after which SNPs were called using UnifiedGenotyper. Finally, a series of quality filters were applied to reduce systematic errors, including mapping quality $(M Q) \geq 20$, quality-by-depth ratio $(\mathrm{QD}) \geq 2$, ReadPosRankSum $\geq-8.0$, depth coverage $(\mathrm{DP}) \geq 3$, probability of strand bias (FS) $\leq 30.0$, and no more than three SNPs within $10 \mathrm{bp}$. SNPs that passed these filters were kept for subsequent analyses.

\section{Identification of orthologues and homologues}

Orthologous gene groups in A. thaliana, A. lyrata, and C. rubella were identified using InParanoid v2.0 [60] with default parameters. MCScanX [61] was used to identify synteny in the three genomes to confirm the orthologous relationships of these genes. Only those gene groups identified by InParanoid and supported as syntenic were considered orthologous. To assess the number of homologues for the five candidate genes with TSP sites, each gene was searched against the representative genomes of 11 green plants using blastp with an $E$-value threshold of $1 \times 10^{-5}$ according to our previous study [62] (Additional file 1: Table S7).

\section{Identification of shared SNPs}

The sequences of all samples for each orthologous gene were aligned using MUSCLE v3.8.31 [63]. Shared biallelic SNPs were counted. The process was repeated for both coding regions and genic regions.

To guarantee the reliability of shared SNPs, further filtering steps were applied to the C. rubella SNPs. The mappability of each 50-bp region in the reference genome of $C$. rubella was assessed using the 50-bp mappability score [64], which counts the number of times each 50 mer maps to the reference $C$. rubella genome, allowing up to two mismatches. SNPs any of whose overlapping 50 mers could be mapped to more than one locations with two or fewer mismatches were excluded.

To determine whether candidate genes fell in regions of $\mathrm{CNV}, \mathrm{CNVs}$ were detected in each species using CNVnator [65] with a bin size of $500 \mathrm{bp}$ and a $P$ value threshold of 0.01. Candidates with normalized read depths $<0.5$ or $>1.5$ were considered as CNVs of deletion or duplication events, respectively. snpEff $\mathrm{v} 4.2$ [66] was used to annotate the variation for each sample, and SNPs were characterized as synonymous_variant (S), missense_variant (M), or intron_variant (intron).

\section{Demographic inference}

To uncover the neutral evolutionary history of the two species, the orthologous fourfold degenerate sites were extracted from the alignments of the respective reference genomes of $A$. thaliana and C. rubella for the coding regions of orthologues. The three codons of an orthologous amino acid from the two species were required to be aligned together (i.e. not separated by gaps). Afterwards, by restricting the analysis to fixed or bi-allelic sites, 2,011,573 orthologous fourfold degenerate sites were finally used to generate the joint SFS of the two species under study. Fastsimcoal2 [30] was then run on the SFS under Model M1 (no gene flow) and M2 (ancient gene flow). The best parameter estimates under each model were obtained from 50 independent runs with a minimum of 100,000 and a maximum of 1,000,000 coalescent simulations as well as $10-40$ cycles of the likelihood maximization algorithm. SFS entries with support from fewer than six SNPs were ignored, as suggested by Excoffier et al. [30]. The 95\% CIs of the best 
parameter estimates were obtained by non-parametric block bootstrapping, as in Malaspinas et al. [67], and each orthologue was considered as a block.

\section{Allelic tree searching}

A sliding window approach was used to scan every valid 100-bp window that covered each shared SNP in the genic regions of the 433 candidate genes with reliable shared coding SNPs for regions of sequences clustering by allele. To be a valid window, the number of effectively aligned sites (A, C, G, or T) must be no less than 95 in each species to minimize gaps or missing bases in the $A$. thaliana SNP matrix. Phylogenetic neighbor-joining tree [68] construction was performed using the Phylip package with default parameters [69] and each tree was checked whether all the samples from one species were clustered together against the other. Trees that failed such clustering became candidate allelic trees and were checked manually, topological robustness was evaluated with 1000 bootstrapping replicates using MEGA v6 [70]. Linkage disequilibrium was evaluated by calculating $r^{2}$ using PLINK [71].

\section{Analysis of PCR-verified candidate regions}

All haplotypes for each candidate region were obtained using DnaSP v5.10.1 [72]. Neighbor-joining trees of the PCR sequences of the candidate regions under balancing selection were constructed using MEGA v6 [70] with the Kimura two-parameter model [68, 73]. Topological robustness was assessed by bootstrapping with 1000 replicates [74].

\section{Ecological analysis}

ADMIXTURE [47] was used to estimate the population structure in the $80 \mathrm{~A}$. thaliana samples [25]. Such analysis was run based on the $1,438,787$ SNPs removing sites ambiguous in any sample from the original SNP matrix; group numbers $(K)$ from 1 to 10 were explored. Finally, $K=2$, with the lowest cross-validation error, was the fittest number. Correlations between the structure groups and allelic types were assessed by chisquare tests.

To reduce the effects of sampling bias, sample records were spatially thinned using the randomization method in the spThin $\mathrm{R}$ package [75]. The "thin" method was used, setting the parameter "thin.par" to be $5 \mathrm{~km}$, namely, every two samples should be at least $5 \mathrm{~km}$ apart.

To investigate the ecological characteristics associated with the loci under ancient balancing selection, ecological niche modeling (ENM) and ecological niche identity tests were performed [49]. ENMTools [76] was used to calculate the niche overlap between the two haplotype groups assessed by Warren's I similarity statistics, ranging from 0 (no overlap) to 1 (identical niches), with 100 pseudo-replicates, according to our previous study [77]. The one-sample $t$-test was employed when comparing the observed $I$ score with the scores for a set of permutations. Different permutation strategies were achieved by modifying the options in ENMTools [76].

\section{Statistical analysis}

All statistical analyses were performed using $\mathrm{R}$ package v3.1.3 [78]. All $P$ values from multiple testing were adjusted using the "fdr" option in the "p.adjust" function in $\mathrm{R}$ [79]. All violin plots and histograms were generated using $\mathrm{R}$ as well.

\section{Additional files}

Additional file 1: Table S1. List of C. rubella accessions included in this study. Table S2. Summary of SNPs called from populations of each species. Table S4. Primers for PCR amplification and sequencing. Table S5. Statistics of the SNPs at CpG and non-CpG sites in the genic regions of 16,014 orthologous genes. Table S6. Simulation results for different demographic models. Table S7. Number of homologous genes for each of the confirmed genes with trans-species polymorphism signals in green plants. Table S10. Correlation between the structure and allelic type in $80 \mathrm{~A}$. thaliana samples for each of the five genes under balancing selection. (DOCX $63 \mathrm{~kb}$ )

Additional file 2: Table S3. Demographic inference results from fastsimcoal2. (XLSX $15 \mathrm{~kb})$

Additional file 3: Figure S1. Allelic trees across the two species based on the 100-bp window around the TSP sites for each of the five genes under balancing selection. All A. thaliana accessions are colored in red and numbered according to the accessions listed on the 1001 Genomes site (http://1001 genomes.org/projects/MPICao2010/index.html); see Additional file 5: Table S8C for details. All C. rubella accessions are shown in black and numbered according to Additional file 1: Table S1. Figure S2. Distribution of the (A) nucleotide diversity $(\pi)$ and (B) MAF values of the simulated neutral sequences of $100 \mathrm{bp}$ under the estimated model in each species. Triangles in different colors in (A) indicate the average values for all qualified windows in the five genes. See Table 1 for the details of each site (labeled TSP-1 to TSP-10). Figure S3. Cross-validation errors for various numbers of clusters (K) in an ADMIXTURE analysis. Figure S4. Geographic distributions of samples of different allelic types for the four genes under long-term balancing selection excluding AT5G38460. (DOCX $5905 \mathrm{~kb}$ )

Additional file 4: Text S1. Coalescence-based simulation to support the candidate TSPs. (DOCX $45 \mathrm{~kb}$ )

Additional file 5: Table S8. Ecological analysis: source data and results. A. The 48 ecological factors used in the analysis and the divergence of the two types of samples for each gene with regard to these factors. In each column of the four genes, the FDR-corrected $P$ values are listed, and the significant ones are highlighted in red. B. Sample size distribution based on the haplotype phasing for each gene before and after thinning of the 1135 genomes. C. Sample information and classification for each gene. "-" indicates that the corresponding sample is not phased properly in the corresponding gene. D. The ecological significance of the four genes. (XLSX $52 \mathrm{~kb}$ )

Additional file 6: Table S9. Structure of the $80 \mathrm{~A}$. thaliana samples. The values for Group 1 and Group 2 indicate the corresponding ancestral fraction, respectively. (XLSX $12 \mathrm{~kb}$ )

\section{Acknowledgements}

We thank the anonymous reviewers for their help improving the manuscript, Detlef Weigel and Daniel Koenig for access to the resequencing datasets of Capsella rubella, and Xing Wang Deng and Guangming He for the Arabidopsis thaliana seeds. We also thank Haipeng Li, Zhe Cai, and Fumin Zhang for their helpful discussions. 


\section{Funding}

This work was supported by the National Natural Science Foundation of China (91231104, 31222006, and 31470331 to YLG; 61103066 to QW) and the 100 Talents Program of the Chinese Academy of Sciences (YLG).

\section{Availability of data and materials}

The genome sequence of ecotype 928 of C. rubella reported in this paper has been deposited in the NCBI Sequence Read Archive (SRA) under accession number SRP066343 [27]. Sequences obtained by PCR have been submitted to GenBank (accession numbers KU233378-KU233484, KY123666KY123684) [38, 39]. The code has been deposited in a public repository under the link: https://github.com/anai1031/TSP/ [80].

\section{Authors' contributions}

YLG conceived the study. QW performed the principal analysis. TSH, ZWL, JFC, YPZ, and YCX performed the ecological and other analyses. XC and TSH performed the validation experiments. QW and YLG wrote the paper with contributions from all other authors. All authors read and approved the final manuscript.

\section{Ethics approval and consent to participate}

Not applicable.

\section{Consent for publication}

Not applicable.

\section{Competing interests}

The authors declare that they have no competing interests.

\section{Publisher's Note}

Springer Nature remains neutral with regard to jurisdictional claims in published maps and institutional affiliations.

\section{Received: 11 July 2017 Accepted: 16 October 2017}

Published online: 15 November 2017

\section{References}

1. Key FM, Teixeira JC, de Filippo C, Andres AM. Advantageous diversity maintained by balancing selection in humans. Curr Opin Genet Dev. 2014;29:45-51.

2. Charlesworth D. Balancing selection and its effects on sequences in nearby genome regions. Plos Genet. 2006;2:379-84.

3. Leffler EM, Gao Z, Pfeifer S, Segurel L, Auton A, Venn O, et al. Multiple instances of ancient balancing selection shared between humans and chimpanzees. Science. 2013;339:1578-82.

4. Barson NJ, Aykanat T, Hindar K, Baranski M, Bolstad GH, Fiske P, et al. Sexdependent dominance at a single locus maintains variation in age at maturity in salmon. Nature. 2015;528:405-8.

5. Ségurel L, Thompson EE, Flutre T, Lovstad J, Venkat A, Margulis SW, et al. The $\mathrm{ABO}$ blood group is a trans-species polymorphism in primates. Proc Natl Acad Sci U S A. 2012;109:18493-8.

6. Chakraborty M, Fry JD. Evidence that environmental heterogeneity maintains a detoxifying enzyme polymorphism in Drosophila melanogaster. Curr Biol. 2016;26:219-23.

7. Dobzhansky T. Genetics and the origin of species. 3rd ed. New York: Columbia University Press; 1951

8. Klein J, Sato A, Nikolaidis N. MHC, TSP, and the origin of species: from immunogenetics to evolutionary genetics. Annu Rev Genet. 2007:41:281-304.

9. Wu J, Saupe SJ, Glass NL. Evidence for balancing selection operating at the het-c heterokaryon incompatibility locus in a group of filamentous fungi. Proc Natl Acad Sci U S A. 1998;95:12398-403.

10. Guo YL, Zhao X, Lanz C, Weigel D. Evolution of the S-locus region in Arabidopsis relatives. Plant Physiol. 2011;157:937-46.

11. Roux C, Pauwels M, Ruggiero MV, Charlesworth D, Castric V, Vekemans X. Recent and ancient signature of balancing selection around the S-locus in Arabidopsis halleri and A. lyrata. Mol Biol Evol. 2013;30:435-47.

12. Bechsgaard JS, Castric V, Charlesworth D, Vekemans X, Schierup MH. The transition to self-compatibility in Arabidopsis thaliana and evolution within S-haplotypes over 10 Myr. Mol Biol Evol. 2006;23:1741-50

13. Nasrallah JB. Recognition and rejection of self in plant reproduction. Science. 2002;296:305-8.
14. Karasov TL, Kniskern JM, Gao L, DeYoung BJ, Ding J, Dubiella U, et al. The long-term maintenance of a resistance polymorphism through diffuse interactions. Nature. 2014;512:436-40.

15. Malaria Genomic Epidemiology Network. A novel locus of resistance to severe malaria in a region of ancient balancing selection. Nature. 2015;526:253-7.

16. Greene JS, Brown M, Dobosiewicz M, Ishida IG, Macosko EZ, Zhang X, et al. Balancing selection shapes density-dependent foraging behaviour. Nature. 2016:539:254-8

17. Fijarczyk A, Babik W. Detecting balancing selection in genomes: limits and prospects. Mol Ecol. 2015;24:3529-45.

18. Gao Z, Przeworski M, Sella G. Footprints of ancient-balanced polymorphisms in genetic variation data from closely related species. Evolution. 2014;69:431-46.

19. Teixeira JC, de Filippo C, Weihmann A, Meneu JR, Racimo F, Dannemann M, et al. Long-term balancing selection in LAD1 maintains a missense transspecies polymorphism in humans, chimpanzees, and bonobos. Mol Biol Evol. 2015;32:1186-96.

20. Novikova PY, Hohmann N, Nizhynska V, Tsuchimatsu T, Ali J, Muir G, et al. Sequencing of the genus Arabidopsis identifies a complex history of nonbifurcating speciation and abundant trans-specific polymorphism. Nat Genet. 2016:48:1077-82.

21. Hohmann N, Wolf EM, Lysak MA, Koch MA. A time-calibrated road map of Brassicaceae species radiation and evolutionary history. Plant Cell. 2015;27:2770-84

22. Brandvain Y, Slotte T, Hazzouri KM, Wright SI, Coop G. Genomic identification of founding haplotypes reveals the history of the selfing species Capsella rubella. PLoS Genet. 2013;9:e1003754.

23. Foxe JP, Slotte T, Stahl EA, Neuffer B, Hurka H, Wright SI. Recent speciation associated with the evolution of selfing in Capsella. Proc Natl Acad Sci USA. 2009;106:5241-5.

24. Guo YL, Bechsgaard JS, Slotte T, Neuffer B, Lascoux M, Weigel D, et al. Recent speciation of Capsella rubella from Capsella grandiflora, associated with loss of self-incompatibility and an extreme bottleneck. Proc Natl Acad Sci U S A. 2009;106:5246-51.

25. Cao J, Schneeberger K, Ossowski S, Gunther T, Bender S, Fitz J, et al. Wholegenome sequencing of multiple Arabidopsis thaliana populations. Nat Genet. 2011:43:956-63.

26. Ågren J, Wang W, Koenig D, Neuffer B, Weigel D, Wright SI. Mating system shifts and transposable element evolution in the plant genus Capsella. BMC Genomics. 2014;15:602

27. Wu Q. Resequencing data for a sample of Capsella rubella. NCBI SRA. 2015; SRP066343.

28. Slotte T, Hazzouri KM, Ågren JA, Koenig D, Maumus F, Guo Y-L, et al. The Capsella rubella genome and the genomic consequences of rapid mating system evolution. Nat Genet. 2013;45:831-5.

29. Hu T, Pattyn P, Bakker EG, Cao J, Cheng JF, Clark RM, et al. The Arabidopsis lyrata genome sequence and the basis of rapid genome size change. Nat Genet. 2011:43:476-81.

30. Excoffier L, Dupanloup I, Huerta-Sánchez E, Sousa VC, Foll M. Robust demographic inference from genomic and SNP data. Plos Genet. 2013;9:e1003905.

31. Li H, Stephan W. Inferring the demographic history and rate of adaptive substitution in Drosophila. Plos Genet. 2006;2:e166.

32. Koch MA, Kiefer M. Genome evolution among cruciferous plants: a lecture from the comparison of the genetic maps of three diploid species - Capsella rubella, Arabidopsis Iyrata subsp. petraea, and A. thaliana. Am J Bot. 2005:92:761-7.

33. Ossowski S, Schneeberger K, Lucas-Lledo Jl, Warthmann N, Clark RM, Shaw $\mathrm{RG}$, et al. The rate and molecular spectrum of spontaneous mutations in Arabidopsis thaliana. Science. 2010;327:92-4.

34. Wiuf C, Zhao K, Innan H, Nordborg M. The probability and chromosomal extent of trans-specific polymorphism. Genetics. 2004;168:2363-72.

35. Tang C, Toomajian C, Sherman-Broyles S, Plagnol V, Guo YL, Hu TT, et al. The evolution of selfing in Arabidopsis thaliana. Science. 2007;317:1070-2.

36. Bomblies K, Yant L, Laitinen RA, Kim ST, Hollister JD, Warthmann N, et al. Local-scale patterns of genetic variability, outcrossing, and spatial structure in natural stands of Arabidopsis thaliana. Plos Genet. 2010;6:e1000890

37. Salomé PA, Bomblies K, Fitz J, Laitinen RAE, Warthmann N, Yant L, et al. The recombination landscape in Arabidopsis thaliana F2 populations. Heredity. 2012:108:447-55.

38. Chen X, Guo YL, Wu Q. The PCR sequences of the candidate TSP genes under balancing selection: AT1G35220, AT2G16570, AT5G38460, and AT5G44000. GenBank. 2015:KU233378-484. 
39. Wu Q, Han TS, Chen X, Li ZW, Zou YP, Guo YL. The PCR sequences of the candidate TSP gene AT4G29360 under balancing selection. GenBank. 2016: KY123666-84

40. Yang Z, Guo G, Zhang M, Liu CY, Hu Q, Lam H, et al. Stable isotope metabolic labeling-based quantitative phosphoproteomic analysis of Arabidopsis mutants reveals ethylene-regulated time-dependent phosphoproteins and putative substrates of constitutive triple response 1 kinase. Mol Cell Proteomics. 2013;12:3559-82.

41. Hung WF, Chen LJ, Boldt R, Sun CW, Li HM. Characterization of Arabidopsis glutamine phosphoribosyl pyrophosphate amidotransferase-deficient mutants. Plant Physiol. 2004;135:1314-23.

42. Narsai $R$, Law SR, Carrie C, Xu L, Whelan J. In-depth temporal transcriptome profiling reveals a crucial developmental switch with roles for RNA processing and organelle metabolism that are essential for germination in Arabidopsis. Plant Physiol. 2011;157:1342-62.

43. Ditt RF, Kerr KF, de Figueiredo P, Delrow J, Comai L, Nester EW. The Arabidopsis thaliana transcriptome in response to Agrobacterium tumefaciens. Mol Plant Microbe Interact. 2006;19:665-81.

44. Pasquet JC, Changenet V, Macadre C, Boex-Fontvieille E, Soulhat C, BouchabkeCoussa O, et al. A Brachypodium UDP-Glycosyltransferase confers root tolerance to deoxynivalenol and resistance to fusarium infection. Plant Physiol. 2016;172:559-74.

45. Dixon DP, Skipsey M, Edwards R. Roles for glutathione transferases in plant secondary metabolism. Phytochemistry. 2010;71:338-50.

46. Guo YL, Fitz J, Schneeberger K, Ossowski S, Cao J, Weigel D. Genome-wide comparison of nucleotide-binding site-leucine-rich repeat-encoding genes in Arabidopsis. Plant Physiol. 2011;157:757-69.

47. Alexander DH, Novembre J, Lange K. Fast model-based estimation of ancestry in unrelated individuals. Genome Res. 2009;19:1655-64.

48. The 1001 Genomes Consortium. 1,135 genomes reveal the global pattern of polymorphism in Arabidopsis thaliana. Cell. 2016;166:481-91.

49. Phillips SJ, Anderson RP, Schapire RE. Maximum entropy modeling of species geographic distributions. Ecol Model. 2006;190:231-59.

50. Schmitz RJ, Schultz MD, Urich MA, Nery JR, Pelizzola M, Libiger $O$, et al. Patterns of population epigenomic diversity. Nature. 2013;495:193-8.

51. Li ZW, Chen X, Wu Q, Hagmann J, Han TS, Zou YP, et al. On the origin of de novo genes in Arabidopsis thaliana populations. Genome Biol Evol. 2016;8: 2190-202.

52. Gloss AD, Nelson Dittrich AC, Goldman-Huertas B, Whiteman NK. Maintenance of genetic diversity through plant-herbivore interactions. Curr Opin Plant Biol. 2013;16:443-50.

53. Fournier-Level A, Korte A, Cooper MD, Nordborg M, Schmitt J, Wilczek AM. A map of local adaptation in Arabidopsis thaliana. Science. 2011:334:86-9.

54. Hancock AM, Brachi B, Faure N, Horton MW, Jarymowycz LB, Sperone FG, et al. Adaptation to climate across the Arabidopsis thaliana genome. Science. 2011;334:83-6

55. Kohyama TI, Omote K, Nishida C, Takenaka T, Saito K, Fujimoto S, et al. Spatial and temporal variation at major histocompatibility complex class IIB genes in the endangered Blakiston's fish owl. Zoological Lett. 2015:1:13.

56. Doyle JJ, Doyle JL. A rapid DNA isolation procedure from small quantities of fresh leaf tissues. Phytochem Bull. 1987;19:11-5.

57. Patel RK, Jain M. NGS QC Toolkit: a toolkit for quality control of next generation sequencing data. PLoS One. 2012;7:e30619.

58. Li H, Durbin R. Fast and accurate short read alignment with Burrows-Wheeler transform. Bioinformatics. 2009:25:1754-60.

59. Danecek P, Auton A, Abecasis G, Albers CA, Banks E, DePristo MA, et al. The variant call format and VCFtools. Bioinformatics. 2011;27:2156-8.

60. Berglund AC, Sjolund E, Ostlund G, Sonnhammer EL. InParanoid 6: eukaryotic ortholog clusters with inparalogs. Nucleic Acids Res. 2008;36:D263-266.

61. Wang Y, Tang H, Debarry JD, Tan X, Li J, Wang X, et al. MCScanX: a toolkit for detection and evolutionary analysis of gene synteny and collinearity. Nucleic Acids Res. 2012;40:e49.

62. Guo YL. Gene family evolution in green plants with emphasis on the origination and evolution of Arabidopsis thaliana genes. Plant J. 2013;73:941-51.

63. Edgar RC. MUSCLE: a multiple sequence alignment method with reduced time and space complexity. BMC Bioinformatics. 2004:5:113.

64. Derrien T, Estelle J, Marco Sola S, Knowles DG, Raineri E, Guigo R, et al. Fast computation and applications of genome mappability. PLoS One. 2012;7:e30377.

65. Abyzov A, Urban AE, Snyder M, Gerstein M. CNVnator: an approach to discover, genotype, and characterize typical and atypical CNVs from family and population genome sequencing. Genome Res. 2011;21:974-84.
66. Cingolani P, Platts A, le Wang L, Coon M, Nguyen T, Wang L, et al. A program for annotating and predicting the effects of single nucleotide polymorphisms, SnpEff: SNPs in the genome of Drosophila melanogaster strain w1118; iso-2; iso-3. Fly (Austin). 2012;6:80-92.

67. Malaspinas A-S, Westaway MC, Muller C, Sousa VC, Lao O, Alves I, et al. A genomic history of Aboriginal Australia. Nature. 2016:538:207-14.

68. Saitou N, Nei M. The neighbor-joining method: a new method for reconstructing phylogenetic trees. Mol Biol Evol. 1987;4:406-25.

69. Feisenstein J. PHYLIP-phylogeny inference package (version 3.2). Cladistics. 1989;5:164-6.

70. Tamura K, Peterson D, Peterson N, Stecher G, Nei M, Kumar S. MEGA5: molecular evolutionary genetics analysis using maximum likelihood, evolutionary distance, and maximum parsimony methods. Mol Biol Evol. 2011;28:2731-9.

71. Purcell S, Neale B, Todd-Brown K, Thomas L, Ferreira MA, Bender D, et al. PLINK: a tool set for whole-genome association and population-based linkage analyses. Am J Hum Genet. 2007;81:559-75.

72. Librado P, Rozas J. DnaSP v5: a software for comprehensive analysis of DNA polymorphism data. Bioinformatics. 2009;25:1451-2.

73. Kimura M. A simple method for estimating evolutionary rate of base substitutions through comparative studies of nucleotide sequences. J Mol Evol. 1980;16:111-20

74. Felsenstein J. Confidence limits on phylogenies: an approach using the bootstrap. Evolution. 1985;39:783-91.

75. Aiello-Lammens ME, Boria RA, Radosavljevic A, Vilela B, Anderson RP. spThin: an $\mathrm{R}$ package for spatial thinning of species occurrence records for use in ecological niche models. Ecography. 2015;38:541-5.

76. Warren DL, Glor RE, Turelli M. ENMTools: a toolbox for comparative studies of environmental niche models. Ecography. 2010;33:607-11.

77. Han TS, Wu Q, Hou XH, Li ZW, Zou YP, Ge S, et al. Frequent introgressions from diploid species contribute to the adaptation of the tetraploid Shepherd's purse (Capsella bursa-pastoris). Mol Plant. 2015;8:427-38.

78. R Core Team. R: A language and environment for statistical computing. Vienna: R Foundation for Statistical Computing; 2014

79. Benjamini $Y$, Hochberg $Y$. Controlling the false discovery rate: a practical and powerful approach to mutliple testing. JR Statis Soc B. 1995;57:289-300.

80. Wu Q. Code for the paper "Long-term balancing selection contributes to adaptation in Arabidopsis and its relatives". GitHub. 2017. https://github. com/anai1031/TSP/.

\section{Submit your next manuscript to BioMed Central and we will help you at every step:}

- We accept pre-submission inquiries

- Our selector tool helps you to find the most relevant journal

- We provide round the clock customer support

- Convenient online submission

- Thorough peer review

- Inclusion in PubMed and all major indexing services

- Maximum visibility for your research

Submit your manuscript at www.biomedcentral.com/submit
) Biomed Central 